\title{
Two diagnoses become one? Rare case report of anorexia nervosa and Cushing's syndrome
}

\author{
This article was published in the following Dove Press journal: \\ Neuropsychiatric Disease and Treatment \\ 28 March 2013 \\ Number of times this article has been viewed
}

\author{
Nadia Sawicka* \\ Maria Gryczyńska* \\ Jerzy Sowiński \\ Monika Tamborska- \\ Zedlewska \\ Marek Ruchała \\ Department of Endocrinology, \\ Metabolism and Internal Medicine, \\ Poznan University of Medical Sciences, \\ Poznan, Poland \\ *These authors contributed equally \\ to this work
}

\begin{abstract}
Hypothalamic-pituitary-adrenal axis impairment in anorexia nervosa is marked by hypercortisolemia, and psychiatric disorders occur in the majority of patients with Cushing's syndrome. Here we report a patient diagnosed with anorexia nervosa who also developed Cushing's syndrome. A 26-year-old female had been treated for anorexia nervosa since she was 17 years old, and also developed depression and paranoid schizophrenia. She was admitted to the Department of Endocrinology, Metabolism, and Internal Medicine with a preliminary diagnosis of Cushing's syndrome. Computed tomography revealed a $27 \mathrm{~mm}$ left adrenal tumor, and she underwent laparoscopic adrenalectomy. She was admitted to hospital 6 months after this procedure, at which time she did not report any eating or mood disorder. This is a rare case report of a patient with anorexia nervosa in whom Cushing's syndrome was subsequently diagnosed. Diagnostic difficulties were caused by the signs and symptoms presenting in the course of both disorders, ie, hypercortisolemia, osteoporosis, secondary amenorrhea, striae, hypokalemia, muscle weakness, and depression.
\end{abstract}

Keywords: anorexia nervosa, Cushing's syndrome, adrenalectomy, osteoporosis

\section{Introduction}

Anorexia nervosa is one of the most common psychiatric diseases among young women. The diagnostic criteria are refusal to maintain weight appropriate for age and height, fear of gaining weight, impaired body image, and amenorrhea. ${ }^{1}$ There are two main types of anorexia nervosa, ie, restrictive and purging, and both have an impact on appetite-regulating hormones. Moreover, they lead to changes in the hypothalamicpituitary-gonadal and hypothalamic-pituitary-adrenal axes, bone loss, and growth hormone resistance. Impairment of the hypothalamic-pituitary-adrenal axis in anorexia nervosa is marked by hypercortisolemia.

On the other hand, psychiatric disorders occur in the majority of patients with Cushing's syndrome. Symptoms include emotional lability, insomnia, and poor memory and concentration. Patients with Cushing's syndrome may also develop more severe psychiatric disturbances, such as depression with suicide attempts, psychosis with delusions and hallucinations, and paranoia. ${ }^{2}$ Here we report a patient diagnosed with anorexia nervosa who developed Cushing's syndrome, and discuss the diagnostic difficulties in such an unusual situation.

\section{Case report}

A 26-year-old woman was admitted to the Department of Endocrinology, Metabolism, and Internal Medicine with a preliminary diagnosis of Cushing's syndrome. She also had 
a history of an eating disorder which started when she was 17 years of age. She avoided meals, induced vomiting, and engaged in excessive physical activity. She also developed secondary amenorrhea and was admitted to a psychiatric ward with a body mass index of $14 \mathrm{~kg} / \mathrm{m}^{2}$. She suffered from purging type anorexia nervosa, diagnosed according to DSM-IV (Diagnostic and Statistical Manual of Mental Disorders, Fourth Edition) criteria. ${ }^{1}$ She did not recover and was hospitalized in psychiatric wards on three subsequent occasions. She was also diagnosed with depression 3 years later, for which treatment with olanzapine and a selective serotonin reuptake inhibitor was unsuccessful.

She gradually became physically inactive, and at the age of 20 years, developed new psychiatric symptoms, staying in bed complaining of exhaustion and not participating in social activities. Her mood was depressed, and she experienced changes in her appetite and body weight. Wide red striae were noted on her abdomen and thighs, resulting from a weight recovery of $9 \mathrm{~kg}$. Her morning serum cortisol level was measured at 8 am (when she was hospitalized in psychiatric ward) and the result was at the upper limit of the normal range. She also failed to maintain personal hygiene, and deterioration in her appearance was noted. A diagnosis of paranoid schizophrenia was established when she was 25 years of age during her most recent hospitalization in the psychiatric ward. Pathological fractures of the left proximal tibia and head of fibula were diagnosed 2 months before admission to the Department of Endocrinology.

The patient was irrational and refused to cooperate during the admitting process, claiming that she suffered from colon cancer. Certain of her inevitable death, she refused to leave her bed. It was impossible to measure the patient's height and weight because of lack of cooperation. Blood pressure was about 140/80 mmHg. On physical examination she was noted to have centrally distributed fat and a moon face. Wide red striae on the abdomen and thighs depressed below the surface of the skin were found. Bruises over the lower extremities, androgenic alopecia, and facial acne were also present.

Laboratory tests revealed hypercortisolemia with loss of the circadian rhythm of cortisol and increased urine free cortisol in a 24-hour urine collection (Table 1). An overnight low-dose (1 mg) dexamethasone suppression test was performed, and the plasma cortisol level determined early on the following morning was $566 \mathrm{nmol} / \mathrm{L}$. The cortisol level measured after a high-dose dexamethasone suppression test was $627 \mathrm{nmol} / \mathrm{L}$. The plasma adrenocorticotropic hormone concentration was suppressed $(2.91 \mathrm{pg} / \mathrm{mL})$. Dexamethasone suppression followed by a corticotropin-releasing hormone stimulation test helped to distinguish between Cushing's
Table I Serum cortisol and urine free cortisol in a 24-hour urine collection

\begin{tabular}{ll}
\hline $\begin{array}{l}\text { Serum cortisol } \\
\text { (nmol/L) }\end{array}$ & $\begin{array}{l}\text { Normal range } \\
(\mathrm{nmol} / \mathrm{L})\end{array}$ \\
\hline $8 \mathrm{am}, 766$ & $171-536$ \\
\hline $6 \mathrm{pm}, 732$ & $64-327$ \\
\hline UFC & $\begin{array}{l}\text { Normal range } \\
\text { (nmol/24 hours) }\end{array}$ \\
\hline (nmol/24 hours) & $100-379$ \\
\hline 3834 & \\
\hline
\end{tabular}

Note: Serum cortisol and urine free cortisol in a 24- hour urine collection (UFC) in the Department of Endocrinology, Metabolism and Internal Medicine, Poznan University of Medical Sciences, Poznan, Poland.

Abbreviation: UFC, urinary free cortisol.

syndrome and a pseudo-Cushing state (Table 2). A $27 \mathrm{~mm}$ left adrenal tumor was localized in a computed tomographic scan of her adrenal glands (Figure 1). Compression fractures of the thoracic vertebrae as a result of osteoporosis were also identified (Figure 2). A diagnosis of adrenocorticotropic hormone-independent Cushing's syndrome was made and the patient was referred for left adrenalectomy. Initially the patient refused surgery, but eventually underwent laparoscopic left adrenalectomy 2 months later. Histopathological examination of the tumor confirmed adrenocortical adenoma, and hydrocortisone replacement therapy was started.

The patient was readmitted to hospital 6 months following her surgery, but without depressive symptoms or deterioration in personal appearance and hygiene as before. Her compliance with treatment was good, her appetite was not impaired, and her body mass index was $23 \mathrm{~kg} / \mathrm{m}^{2}$. There were no episodes of self-induced purging after meals reported by the patient or her mother. Her olanzapine dose was decreased and discontinuation of treatment was supervised by a psychiatrist. A normal menstrual cycle returned spontaneously 4 months after adrenalectomy, and since then, the patient's menses have been regular. At her most recent follow-up visit, dual-energy x-ray absorptiometry was performed and secondary osteoporosis was confirmed (Z-score at lumbar spine -3.78; Z-score at femoral neck -3.09).

Table 2 Serum cortisol concentrations on low-dose dexamethasone suppression test, high-dose dexamethasone suppression test, and dexamethasone-CRH test

\begin{tabular}{lll}
\hline Test & $\begin{array}{l}\text { Serum cortisol } \\
\text { (nmol/L) }\end{array}$ & $\begin{array}{l}\text { Normal response } \\
\text { (nmol/L) }\end{array}$ \\
\hline LDDS & 566 & $<50$ \\
HDDS & 627 & \\
D-CRH & 629 & $<38.6$ \\
\hline
\end{tabular}

Abbreviations: D-CRH, dexamethasone-corticotropin-releasing hormone; HDDS, high-dose dexamethasone suppression test; LDDS, low-dose dexamethasone suppression test. 


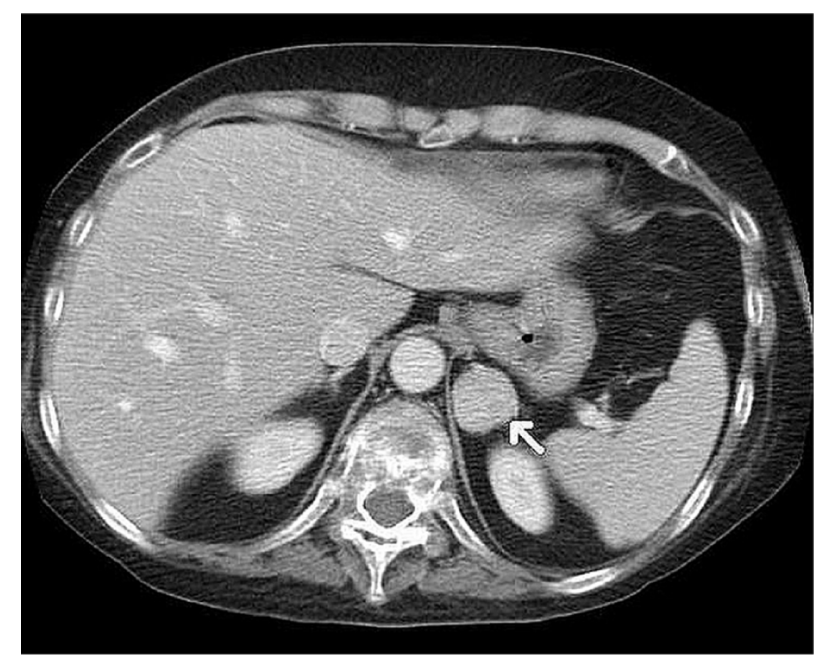

Figure I Computed tomographic scan showing a $27 \mathrm{~mm}$ lesion in the left adrenal gland.

\section{Discussion}

This report of an association between anorexia nervosa and Cushing's syndrome is most unusual. Interestingly, surgical treatment of Cushing's syndrome led to resolution of anorexia nervosa in our patient, so it is possible that Cushing's syndrome was responsible for her eating disorder. However, the diagnosis was difficult to reach because of the signs and symptoms presenting in the course of both disorders, ie, hypercortisolemia, osteoporosis, secondary amenorrhea, striae, hypokalemia, muscle weakness, and depression.

Hypercortisolemia associated with anorexia nervosa is well recognized, although an increased cortisol level is not found in anorexic women as often as in patients with Cushing's syndrome. Increased daily cortisol secretion and

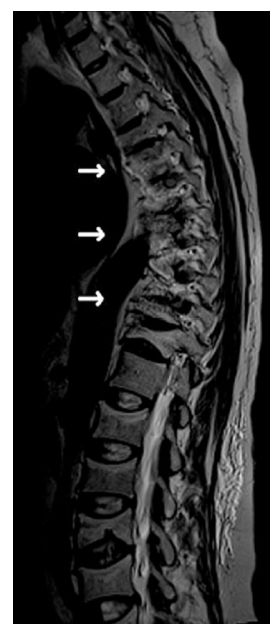

Figure 2 Magnetic resonance imaging showing compression fractures of thoracic vertebral bodies at T4, T7, TI0, and TII.

Note: The spinal cord remains intact. blunted inhibition by low-dose dexamethasone has been reported in many studies of adolescents and adult women with anorexia nervosa, ${ }^{3-5}$ and increased 24-hour urine free cortisol levels have been observed in adult anorexic women, ${ }^{4}$ but not in most studies of adolescent girls with the disorder. However, comparison of urine free cortisol standardized for surface area and creatinine has shown higher cortisol levels in adolescent patients with anorexia nervosa than in their healthy counterparts. ${ }^{5}$ Salivary cortisol levels are also higher in patients with anorexia nervosa than in normal controls, and a statistically significant increase in salivary cortisol concentrations has been observed in anorexic women throughout the day and after dexamethasone suppression. ${ }^{4}$ In addition, lower salivary cortisol levels were found in subjects who underwent psychopharmacological treatment in contrast with untreated anorexic patients. ${ }^{4}$ Hypercortisolemia in patients with anorexia nervosa is a result of increased frequency of bursts of cortisol secretion. ${ }^{4}$ Corticotropin-releasing hormone seems to be the main mechanism responsible for elevated cortisol levels. The concentration of corticotropin-releasing hormone in cerebrospinal fluid in patients with anorexia nervosa has been found to be higher than in controls, ${ }^{6}$ and weight gain was followed by decreased pulsatile secretion of cortisol in adolescents with anorexia nervosa. ${ }^{4}$

In our patient, the cortisol level was relatively higher than in anorexic women, and serum cortisol determined after dexamethasone suppression followed by a corticotropinreleasing hormone stimulation test indicated Cushing's syndrome as a cause of her hypercortisolemia.

It is not known why patients with anorexia nervosa do not develop cushingoid features. Invitti et al showed that the number and affinity of glucocorticoid receptors were comparable in patients with anorexia nervosa and those with active Cushing's disease. ${ }^{7}$ In addition, a decreased plasma adrenocorticotropic hormone response to corticotropinreleasing hormone in underweight patients with anorexia nervosa argues against glucocorticoid resistance. ${ }^{8}$ Lack of a substrate for developing cushingoid features may play a role in patients with anorexia nervosa. Moreover, central redistribution of fat during weight gain has been described in patients with anorexia nervosa who have recovered. ${ }^{9}$ Our patient presented with increased truncal fat and wide red striae from weight recovery in a psychiatric hospital.

The link between anorexia nervosa and hypercortisolemia is unclear as yet. The glucose concentration is the most significant predictive factor for pulsatile secretion of cortisol in anorexia nervosa. ${ }^{5}$ Moreover, in contrast with healthy controls, cortisol is not suppressed by a glucose load in 
patients with anorexia nervosa. ${ }^{5,10}$ This suggests that elevation of cortisol may protect against hypoglycemia, thus acting as a counter-regulatory hormone.

Our patient suffered from severe osteoporosis, with pathological fractures of thoracic vertebrae, tibia, and fibula. Exposure to high-dose glucocorticoids has a deleterious impact on the skeleton, and osteopenia and osteoporosis are a significant problem for patients with Cushing's syndrome. Low bone mineral density is also a common complication of anorexia nervosa, as reported by many studies. ${ }^{11-13}$ One population-based retrospective study reported that fractures of the hip, spine, and forearm occurred on average, 24, 25, and 38 years, respectively, after a diagnosis of anorexia nervosa. ${ }^{14}$ Our patient suffered pathological fractures about 10 years after diagnosis of anorexia nervosa, suggesting additional mechanisms of bone loss. The severe osteoporosis documented in our patient had its origin in both hypercortisolemia and anorexia nervosa. Long-term immobilization probably also contributed to her low bone mineral density. The patient's refusal to leave her bed was interpreted as a symptom of depression, but could have been simply the result of severe pain caused by compression fractures of the thoracic vertebrae. Adolescent girls with anorexia nervosa fail to achieve peak bone mass, ${ }^{15}$ and the onset of the disorder occurred in our patient before acquisition of peak bone mass. Multiple factors, including low concentrations of estrogens, androgens, insulin-like growth factor-1, and leptin, as well as hypercortisolemia, contribute to bone loss in patients with anorexia nervosa. Serum calcium levels and vitamin D intake in affected patients are comparable with those in healthy controls, suggesting that these factors do not have a crucial role in bone loss in patients with anorexia nervosa. ${ }^{16}$ The high serum cortisol level in our patient would have contributed to her bone loss. Bone mineral density and Z-score at the spine have been shown to be lower in patients with anorexia nervosa than in women with hypothalamic amenorrhea. ${ }^{17}$ Earlier research indicates that cortisol levels correlate negatively with bone mineral density in women with anorexia nervosa. Misra et al found an inverse correlation between cortisol concentration and markers of bone formation (C-terminal propeptide of type 1 procollagen and osteocalcin) in patients with anorexia nervosa. ${ }^{5}$ In contrast, the study mentioned above did not find any correlation between cortisol levels and markers of bone formation in healthy controls. Markers of bone resorption correlate weakly with plasma cortisol levels in both patients with anorexia nervosa and healthy controls. In addition, many studies indicate that estrogen replacement or oral contraceptive therapy is ineffective when used to treat osteoporosis in patients with anorexia nervosa. ${ }^{18}$ This finding suggests that high cortisol levels in patients with anorexia nervosa suppress bone formation and may be considered to be a potential mediator of bone loss.

Patients with anorexia nervosa and those with Cushing's syndrome have high rates of comorbid depression. ${ }^{19,20}$ Our patient developed depressive symptoms in the course of anorexia nervosa and underwent pharmacological treatment, but her response to therapy was unsatisfactory. Hypercortisolemia in Cushing's syndrome is considered to be the likely cause of concomitant depression. Depression is recognized to be a potential side effect of exogenous glucocorticoids, and some studies have shown that antiglucocorticoid therapy may improve symptoms in depressed patients with hypercortisolemia. ${ }^{21,22}$ However, the relationship between hypercortisolemia and depression remains unclear. A correlation between cortisol concentration and scores for depressive symptoms has been found, ${ }^{17}$ and between the Hamilton Rating Scale for Depression score and 12-hour overnight serum cortisol levels. Hypercortisolemia may cause psychiatric disturbances in anorexia nervosa. On the other hand, anorexia nervosa may lead to mood disorders following an increase in cortisol concentration. This association is not clearly established and requires further investigation.

Amenorrhea is one of the diagnostic criteria for anorexia nervosa in premenopausal females. Normal menstrual function is disrupted in patients with anorexia nervosa as a result of hypothalamic disturbances followed by suppression of luteinizing hormone. ${ }^{26}$ Many recent studies ${ }^{23-25,27}$ have attempted to identify regulators of the gonadotropin-releasing hormone pulsatility pattern, such as leptin and kisspeptin. Most patients with anorexia nervosa resume normal menstruation after recovery of body weight. ${ }^{28}$ Hypoestrogenemia and hypoandrogenemia in patients with anorexia nervosa resulted from hypogonadotropic hypogonadism. However, adrenal androgen concentrations are comparable with those in healthy controls. ${ }^{29}$ In addition, androgen deficiency in anorexia nervosa may contribute to depression, with an inverse association found between androgen concentration and depression score. ${ }^{29}$ Secondary amenorrhea is a common menstrual irregularity in women with Cushing's syndrome, and is believed to result from hypercortisolemia and decreased pulsatility of gonadotropin-releasing hormone. ${ }^{27,30}$ Our patient resumed menstruation 4 months after adrenalectomy.

Our patient also experienced hypokalemia, which may have been caused by both repeated vomiting and hypercortisolemia due to Cushing's syndrome. Muscle weakness was her main complaint, and both hypercortisolemia and hypokalemia could have contributed to this problem. 
To the authors' knowledge, there is only one previous case report of an association between Cushing's syndrome and anorexia nervosa, published in $1965 .{ }^{31}$ The possibility that hormonal disturbances may be associated with psychiatric disorders should be borne in mind, and the differential diagnosis requires a multifaceted approach.

\section{Disclosure}

The authors report no conflicts of interest in this work.

\section{References}

1. Diagnostic and Statistical Manual of Mental Disorders, Fourth Edition. Washington, DC: American Psychiatric Association; 1994.

2. Pereira AM, Tiemensma J, Romijn JA. Neuropsychiatric disorders in Cushing's syndrome. Neuroendocrinology. 2010;92 Suppl 1:65-70.

3. Doerr P, Fichter M, Pirke KM, Lund R. Relationship between weight gain and hypothalamic pituitary adrenal function in patients with anorexia nervosa. J Steroid Biochem. 1980;13:529-537.

4. Putignano P, Dubini A, Toja P, et al. Salivary cortisol measurement in normal- weight, obese and anorexic women: comparison with plasma cortisol. Eur J Endocrinol. 2001;145:165-171.

5. Misra M, Miller KK, Almazan C, et al. Alterations in cortisol secretory dynamics in adolescent girls with anorexia nervosa and effects on bone metabolism. J Clin Endocrinol Metab. 2004;89:4972-4980.

6. Hotta M, Shibasaki T, Maruda A, et al. The responses of plasma adrenocorticotropin and cortisol to corticotropin-releasing hormone $(\mathrm{CRH})$ and cerebrospinal fluid immunoreactive $\mathrm{CRH}$ in anorexia nervosa patients. J Clin Endocrinol Metab. 1986;62:319-324.

7. Invitti C, Redaelli G, Baldi G, Cavagnini F. Glucocorticoid receptors in anorexia nervosa and Cushing's disease. Biol Psychiatry. 1999;45:1467-1471.

8. Gold P, Gwirtsman H, Avgerinos P, et al. Abnormal hypothalamicpituitary-adrenal function in anorexia nervosa. Pathophysiologic mechanisms in underweight and weight-corrected patients. $N$ Engl J Med. 1986;314:1335-1342.

9. Grinspoon S, Thomas L, Miller K, Pitts S, Herzog D, Klibanski A. Changes in regional fat redistribution and the effects of estrogen during spontaneous weight gain in women with anorexia nervosa. Am J Clin Nutr. 2001;73:865-869.

10. Tamai H, Kiyohara K, Mukuta T, et al. Responses of growth hormone and cortisol to intravenous glucose loading test in patients with anorexia nervosa. Metabolism. 1991;40:31-34.

11. Miller KK, Grinspoon SK, Ciampa J, Hier J, Herzog D, Klibanski A. Medical findings in outpatients with anorexia nervosa. Arch Intern Med. 2005;165:561-566.

12. Grinspoon S, Thomas E, Pitts S, et al. Prevalence and predictive factors for regional osteopenia in women with anorexia nervosa. Ann Intern Med. 2000;133:790-794.

13. Winston AP, Alwazeer AE, Bankart MJ. Screening for osteoporosis in anorexia nervosa: prevalence and predictors of reduced bone mineral density. Int J Eat Disord. 2008;41:284-287.

14. Lucas AR, Melton LJ, Crowson CS, O'Fallon WM. Long-term fracture risk among women with anorexia nervosa: a population-based cohort study. Mayo Clin Proc. 1999;74:972-977.

Neuropsychiatric Disease and Treatment

\section{Publish your work in this journal}

Neuropsychiatric Disease and Treatment is an international, peerreviewed journal of clinical therapeutics and pharmacology focusing on concise rapid reporting of clinical or pre-clinical studies on a range of neuropsychiatric and neurological disorders. This journal is indexed on PubMed Central, the 'PsycINFO' database and CAS.
15. Soyka LA, Misra M, Frenchman A, et al. Abnormal bone mineral accrual in adolescent girls with anorexia nervosa. J Clin Endocrinol Metab. 2002;87:4177-4185.

16. Hadigan CM, Anderson EJ, Miller KK, et al. Assessment of macronutrient and micronutrient intake in women with anorexia nervosa. Int J Eat Disord. 2000;28:284-292.

17. Lawson EA, Donoho D, Miller KK, et al. Hypercortisolemia is associated with severity of bone loss and depression in hypothalamic amenorrhea and anorexia nervosa. J Clin Endocrinol Metab. 2009;94:4710-4716.

18. Sim LA, McGovern L, Elamin MB, Swiglo BA, Erwin PJ, Montori VM. Effect on bone health of estrogen preparation in premenopausal women with anorexia nervosa: a systematic review and meta-analysis. Int J Eat Disord. 2010;43:218-225.

19. Pollice C, Kaye WH, Greeno CG, Weltzin TE. Relationship of depression, anxiety, and obsessionality to state of illness in anorexia nervosa. Int J Eat Disord. 1997;21:367-376.

20. Hudson JI, Hudson MS, Griffing GT, Melby JC, Pope HG Jr. Phenomenology and family history of affective disorder in Cushing's disease. Am J Psychiatry. 1987;144:951-953.

21. Wolkowitz OM, Reus VI, Chan T, et al. Antiglucocorticoid treatment of depression: double-blind ketoconazole. Biol Psychiatry. 1999;45: 1070-1074.

22. Gallagher P, Malik N, Newham J, Young AH, Ferrier IN, Mackin P. Antiglucocorticoid treatments for mood disorders. Cochrane Database Syst Rev C. 2008;23:1. CD005168 doi: 10.1002/14651858.CD005168. pub2.

23. Chan JL, Mantzoros CS. Role of leptin in energy-deprivation states: normal human physiology and clinical implications for hypothalamic amenorrhoea and anorexia nervosa. Lancet. 2005;366:74-85.

24. Fernandez-Fernandez R, Martini AC, Navarro VM, et al. Novel signals for the integration of energy balance and reproduction. Mol Cell Endocrinol. 2006;254-255:127-132.

25. Kumar S, Kaur G. Intermittent fasting dietary restriction regimen negatively influences reproduction in young rats:a study of hypothalamohypophysial-gonadal axis. PLoS One. 2013;8(1):e52416. doi: 10.1371/ journal.pone.0052416.

26. Boyar RM, Katz J, Finkelstein JW, et al. Anorexia nervosa. Immaturity of the 24- hour luteinizing hormone secretory pattern. $N$ Engl J Med. 1987;291:861-865.

27. Penezic Z, Zarkovic M, Vujović S, et al. Gonadotropin pulsatility in Cushing's syndrome compared with polycystic ovary syndrome. Gynecol Endocrinol. 2005;20:150-154.

28. Golden NH, Jacobson MS, Schebendach J, Solanto MV, Hertz SM, Shenker IR. Resumption of menses in anorexia nervosa. Arch Pediatr Adolesc Med. 1997;151:16-21.

29. Miller KK, Wexler TL, Zha AM, et al. Androgen deficiency: association with increased anxiety and depression symptom severity in anorexia nervosa. J Clin Psychiatry. 2007;68:959-965.

30. Lado-Abeal J, Rodriguez-Arnao J, Newell-Price JD, et al. Menstrual abnormalities in women with Cushing's disease are correlated with hypercortisolemia rather than raised circulating androgen levels. J Clin Endocrinol Metab. 1998;83:3083-3088.

31. Black MM, Hall R, Kay DWK, Kilborn JR. Anorexia nervosa in Cushing's syndrome. J Clin Endocrinol Metab. 1965;25:1030-1033.

The manuscript management system is completely online and includes a very quick and fair peer-review system, which is all easy to use. Visit http://www.dovepress.com/testimonials.php to read real quotes from published authors. 